\title{
Early Error Detection Strategy for Full-Duplex Communication in Wireless Sensor Networks
}

\author{
Tae-Yoon Kim, Dongwan Kim, ${ }^{1}$ Jung-Hyok Kwon, ${ }^{2}$ and Eui-Jik Kim ${ }^{2 *}$ \\ Department of Electrical Engineering, Korea University, \\ 145 Anam-ro, Seongbuk-gu, Seoul 02841, South Korea \\ ${ }^{1}$ Department of Electronic Engineering, Dong-A University, \\ 37 Nakdong-daero 550beon-gil, Saha-gu, Busan 49315, South Korea \\ ${ }^{2}$ Department of Convergence Software, Hallym University, \\ 1 Hallymdaehak-gil, Chuncheon-si, Gangwon-do, 24252 South Korea
}

(Received March 2, 2016; accepted March 10, 2017)

Keywords: full-duplex, Internet of Things, spectral efficiency, traffic relaying, wireless sensor networks

Full-duplex (FD) communication is a promising technology for increasing the wireless capacity of wireless sensor networks (WSNs) for Internet of Things (IoT) services. However, the theoretical throughput gain of FD is difficult to achieve under erroneous channel conditions. Given this problem, we presents an early error detection strategy (EEDS) to maximize the spectral efficiency of the FD relaying (FDR) system in WSNs. To reduce frame retransmission due to bit errors, the EEDS divides the entire medium access control (MAC) service data unit (MSDU) of the frame into several fragments and separately inspects each of them on the receiver side. To verify the effectiveness of EEDS, simulations were conducted under various conditions with variations in bit error rate, the number of fragments, and the size of the MSDU. The results show that the throughput and delay performance of the EEDS can be significantly improved under erroneous channel conditions.

\section{Introduction}

Currently, the wireless sensor network (WSN) is considered a key technical component of the Internet of Things (IoT) owing to its data-gathering capability. Unlike traditional WSNs, the WSNs used in IoT services require a comparatively high throughput performance to support various target objects and their increasing sensing resolution of collected data. In this regard, full-duplex (FD) communication is anticipated to satisfy this bandwidth requirement because of its outstanding spectral efficiency. The recent advances in signal processing and antenna technologies enable sensors to transmit and receive data simultaneously on the same channel through the use of the self-interference cancellation (SIC) technique. ${ }^{(1)}$

This two-way communication ideally doubles the wireless capacity compared with half-duplex (HD) communication. However, in real environments, the throughput gain of FD communications is inevitably degraded owing to factors such as medium access control (MAC) overheads and frame corruption. ${ }^{(2)}$ Therefore, many studies have been conducted to enhance the efficiency of FD communications with the goal of maximizing throughput performance. Jain et al. proposed a

${ }^{*}$ Corresponding author: e-mail: ejkim32@hallym.ac.kr http://dx.doi.org/10.18494/SAM.2017.1565 
centralized access protocol that transmits a busy-tone signal to solve the hidden node problem of wireless networks. ${ }^{(3)}$ Sen et al. proposed carrier sense multiple access with collision notification (CSMA/CN) using SIC. ${ }^{(4)}$ When a received frame collides with another frame, the receiver immediately notifies the senders of the collision.

In this paper, we focus on a three-device FD relaying (FDR) system under erroneous channel conditions. Frame retransmission due to bit errors is the main factor that degrades the spectral efficiency of an FDR system. Therefore, to mitigate the impact of frame retransmission on the relaying system and to maximize the efficiency of FD communication, we propose an early error detection strategy (EEDS) that divides a frame into multiple fragments and checks the errors in each fragment. If an error is detected in any fragment, the relaying device stops forwarding the frame and sends a negative-acknowledgement (NACK) to the source device for fast retransmission. We evaluated the performance of the EEDS in simulations, and the results show that the EEDS improves the end-to-end performance of the relaying path compared with the legacy FDR system.

\section{System Model}

In general, FD communications can be classified into two types in accordance with the network topology: 1) bidirectional FD and 2) relaying FD. As shown in Fig. 1(a), the former uses symmetric traffic flow: $\boldsymbol{A}$ sends frames to $\boldsymbol{B}$, and $\boldsymbol{B}$ sends frames to $\boldsymbol{A}$ in the same time slots. The latter, shown in Fig. 1(b), uses asymmetric traffic flow with three devices: $\boldsymbol{R}$ acts as a relay device for the single flow of data being sent from source device $\boldsymbol{S}$ to destination device $\boldsymbol{D}$. Unlike the bidirectional FD, the relaying FD has a transmission order. The transmission from $S$ to $\boldsymbol{R}$ is referred to as a primary transmission, while the transmission from $\boldsymbol{R}$ to $\boldsymbol{D}$ is a secondary transmission. In the relaying FD, $\boldsymbol{R}$ can initiate the forwarding of a frame toward $\boldsymbol{D}$ immediately after reading the MAC header of the frame from $S$.

\section{Early Error Detection Strategy}

\subsection{Early error detection}

In EEDS, the MAC service data unit (MSDU) within the frame is divided into multiple fragments (i.e., $\mathrm{MSDU}_{1}$ to $\mathrm{MSDU}_{N}$ ), and additional frame check sequences (FCSs) are added at the tail of each fragment, as shown in Fig. 2. The FCSs are inspected with their fragments at the receiver in the order of reception. The number of fragments can be varied by the source device $(\boldsymbol{S})$. In addition, to notify and control the fragments, two bytes of additional control overhead (i.e., FCS

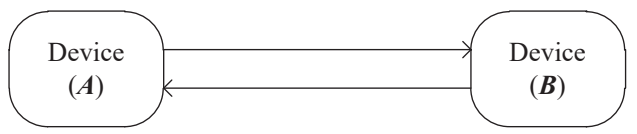

(a)

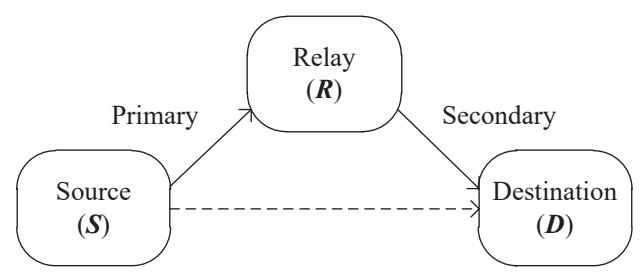

(b)

Fig. 1. Network topologies of FD communications: (a) bidirectional FD and (b) relaying FD. 


\begin{tabular}{|c|c|c|c|c|c|c|c|c|}
\hline \multicolumn{2}{|c|}{ MAC header } & \multicolumn{7}{|c|}{ MSDU ( $N$ fragments $)+$ FCSs } \\
\hline Header & \begin{tabular}{|c|c} 
FCS \\
Control
\end{tabular} & $\mathrm{MSDU}_{1}$ & FCS & $\mathrm{MSDU}_{2}$ & FCS & ... & $\operatorname{MSDU}_{N}$ & FCS \\
\hline $30 \mathrm{~B}$ & $2 \mathrm{~B}$ & $k \mathrm{~B}$ & $4 \mathrm{~B}$ & $k \mathrm{~B}$ & $4 \mathrm{~B}$ & & $k \mathrm{~B}$ & $4 \mathrm{~B}$ \\
\hline
\end{tabular}

Fig. 2. EEDS frame format.

control field) are included in the MAC header.

\subsection{Transmission scheduling}

In EEDS, every fragment is separated with FD interframe space (FIFS) during transmissions to help the receiver distinguish them. If an error is detected on any fragment by checking the FCS at the receiver, the receiver immediately stops forwarding the frame (when the receiver is a relay device, $\boldsymbol{R}$ ) and broadcasts a unique bit array to inform NACK. If $\boldsymbol{S}$ receives NACK for the $k$ th fragment among $N$ fragments, it means that the transmissions up to the $(k-1)$ th fragment were successful and $\boldsymbol{S}$ can restart the transmission from the $k$ th fragment. For the retransmission, $\boldsymbol{S}$ waits for FIFS from the reception of NACK and starts to transmit the remaining fragments with a new header. In this manner, if all fragments in the frame are correctly received, the frame transmission is finished with the reception of an acknowledgement (ACK).

Figure 3 shows examples of frame transmissions of EEDS in the time domain with two errordetection scenarios. Figure 3(a) depicts the operation of error detection on $\boldsymbol{R}$. When $\boldsymbol{R}$ detects an error in the second fragment, it stops forwarding data and broadcasts NACK to $\boldsymbol{S}$ and $\boldsymbol{D}$. Then $\boldsymbol{D}$ waits for the retransmission of the second fragment from $\boldsymbol{S}$.

Figure 3(b) shows an example of error detection on $\boldsymbol{D}$. When $\boldsymbol{D}$ detects an error in the second fragment, it immediately sends NACK back to $\boldsymbol{R}$; then, $\boldsymbol{R}$ relays the NACK to $\boldsymbol{S}$ as soon as the NACK reception is complete. In the FDR system, the timing adjustment for frame transmissions is critical to avoid a collision between the primary and secondary transmissions. Note that $\boldsymbol{S}$ cannot know the occurrence of error at the secondary transmission until the completion of the NACK relay. Thus, if FIFS is too short, $\boldsymbol{S}$ may start to transmit the third fragment while $\boldsymbol{R}$ is receiving the NACK from $\boldsymbol{D}$, resulting in a collision between the NACK and the third fragment at $\boldsymbol{R}$. To address this issue, we make FIFS longer than the sum of the transmission times for the MAC header and NACK.

\section{Performance Evaluation}

In this section, we evaluate the performance of EEDS in the FDR system in terms of the endto-end throughput and the delay on the relaying path using experimental simulations. Although the EEDS is designed to enhance the FDR system under erroneous channel conditions, it also has additional control overheads. To investigate the tradeoffs, we compare the performance of the EEDS with the legacy FDR system in terms of the variations in the bit error rate (BER). In addition, the effects of frame size and the number of fragments on the performance of EEDS are discussed. 


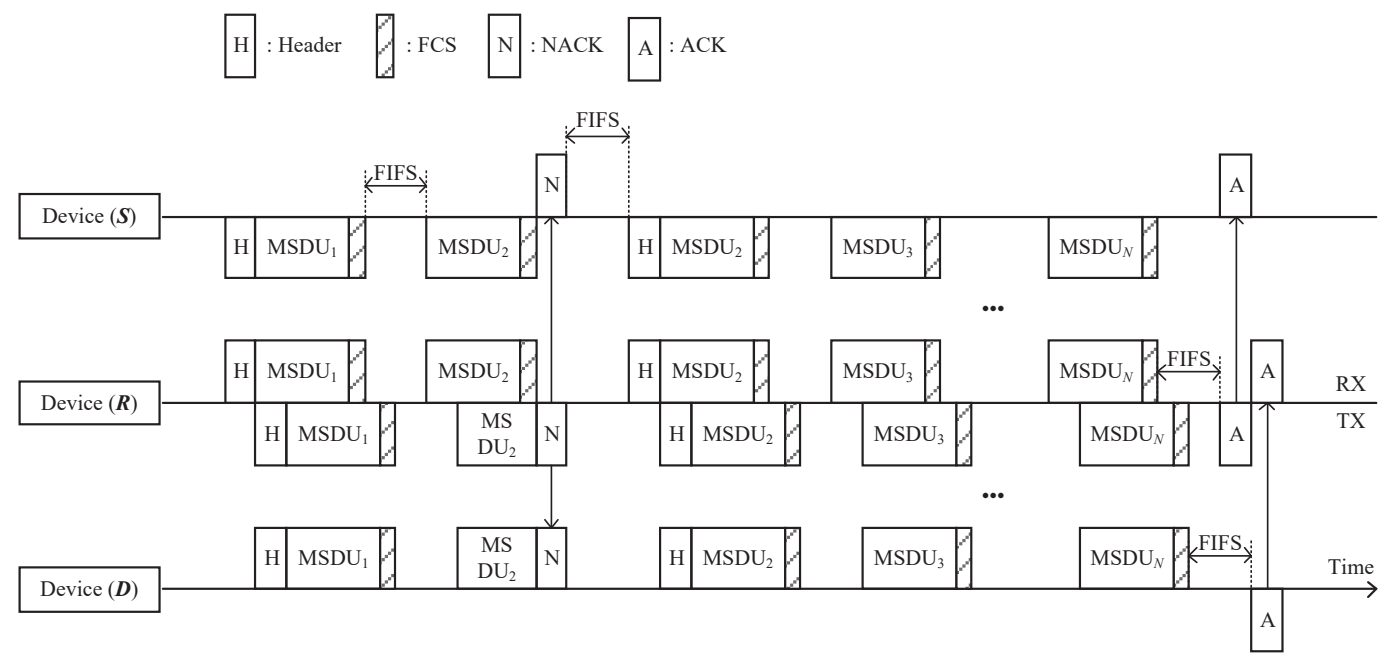

(a)

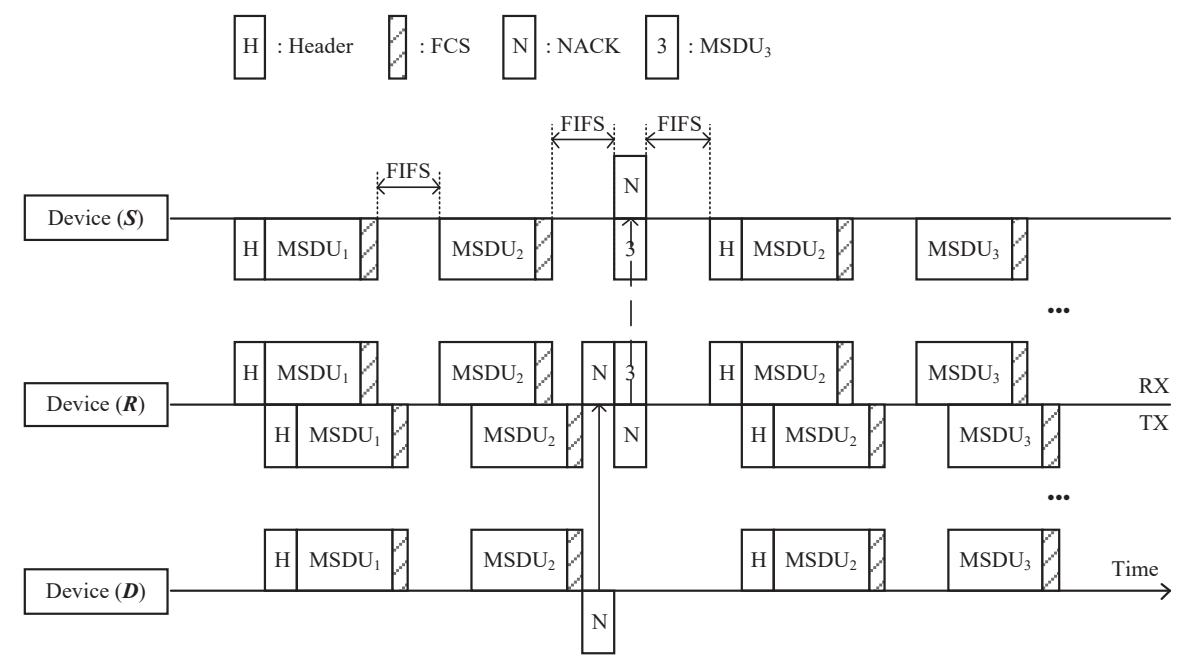

(b)

Fig. 3. Examples of frame transmissions in EEDS: (a) Error detection on $\boldsymbol{R}$ device and (b) error detection on $\boldsymbol{D}$ device.

\subsection{Simulation setup}

A source device with FDR is assumed to transmit the entire frame and then wait for the ACK frame. If the source device does not receive the ACK during the timeout period, it concludes an error and tries to retransmit the entire frame. The simulation parameters used in the performance evaluation are listed in Table 1 . The bit errors are assumed to occur independently for each bit within the frame; thus the error probability of the frame is given as

$$
p_{\text {frame }}=1-\left(1-p_{e}\right)^{m},
$$

where $p_{e}$ is the BER and $m$ is the number of payload bits in the MSDU. This equation is commonly applied to the fragments of EEDS. 
Table 1

Simulation parameters.

\begin{tabular}{lc}
\hline Parameter & Value \\
\hline FIFS & $30 \mu \mathrm{s}$ \\
ACK frame & $14 \mathrm{~B}$ \\
NACK bit array & $2 \mathrm{~B}$ \\
\hline
\end{tabular}

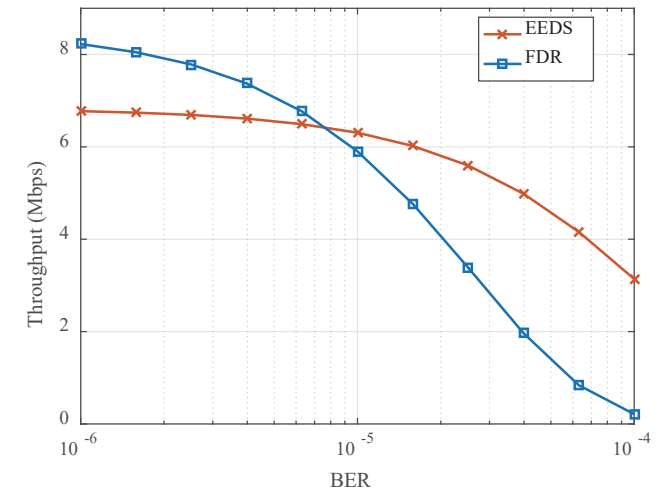

(a)

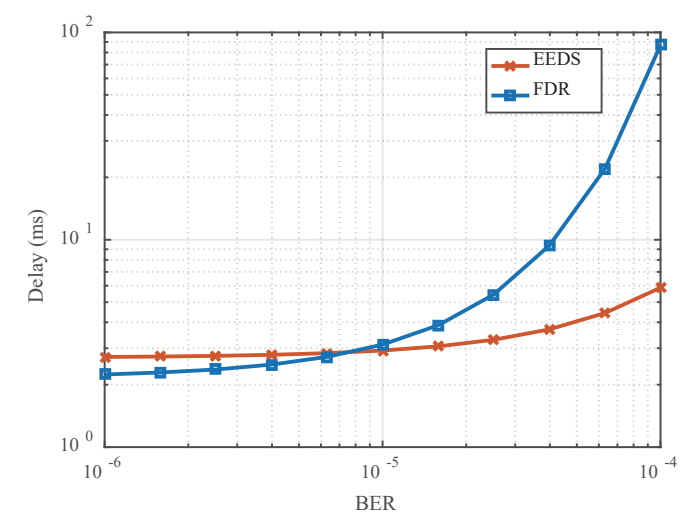

(b)

Fig. 4. (Color online) End-to-end performance (MSDU = $2304 \mathrm{~B}, N=5$ ): (a) average throughput and (b) average delay.

\subsection{Simulation result}

Figure 4 shows the end-to-end performance of EEDS and FDR in terms of the average throughput and delay for an MSDU size of $2304 \mathrm{~B}$ and a number of EEDS fragments $(N)$ of 5 . In Fig. 4(a), the throughput of the FDR under robust channel conditions (when the BER is around $10^{-6}$ ) is higher than that of EEDS, as expected, because the control overhead of EEDS degrades the end-to-end throughput under such conditions. However, when the FDR system operates under channel conditions with errors (when the BER is around $10^{-5}$ ), the EEDS overcomes its additional overhead, and the throughput difference between EEDS and FDR increases as the BER increases. Figure 4(b) shows the average delay. In the figure, when BER is larger than $10^{-5}$, the average delay of FDR starts to exponentially increase, and the difference between FDR and EEDS also increases. EEDS maintains a low error probability during the transmission of the fragments, thereby reducing the time wasted owing to the retransmissions.

Figure 5 shows the throughput ratio for EEDS and FDR, which is obtained by dividing the throughput of EEDS by that of FDR and indicates the efficiency of EEDS. In Fig. 5(a), the more fragments of MSDU are generated, the greater the efficiency of the FDR system under channel conditions with errors. This is because the frame error probability of EEDS is reduced by the increase in the number of fragments. When the BER is $10^{-4}$, EEDS achieves 17 -times better throughput than FDR with $N=6$. Figure 5(b) shows the throughput ratio as a function of MSDU size. If the MSDU size increases, its transmission is more likely to be corrupted owing to frequent bit errors. Therefore, the spectral efficiency of EEDS increases as the MSDU size increases. Specifically, EEDS outperforms FDR by $3400 \%$ when the MSDU size is $3200 \mathrm{~B}$. 


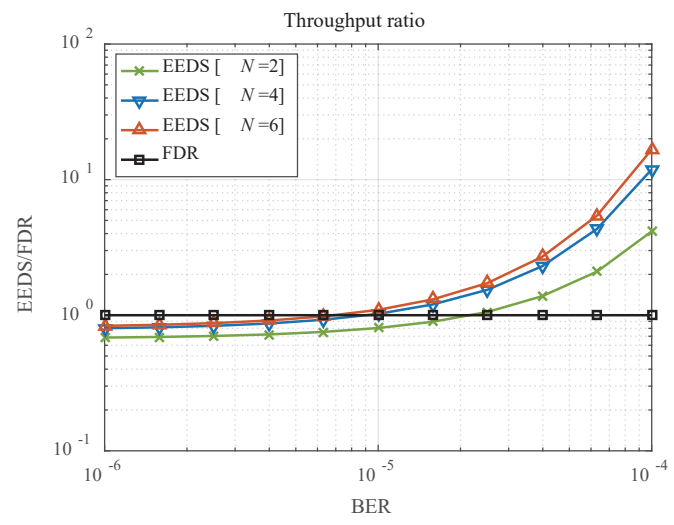

(a)

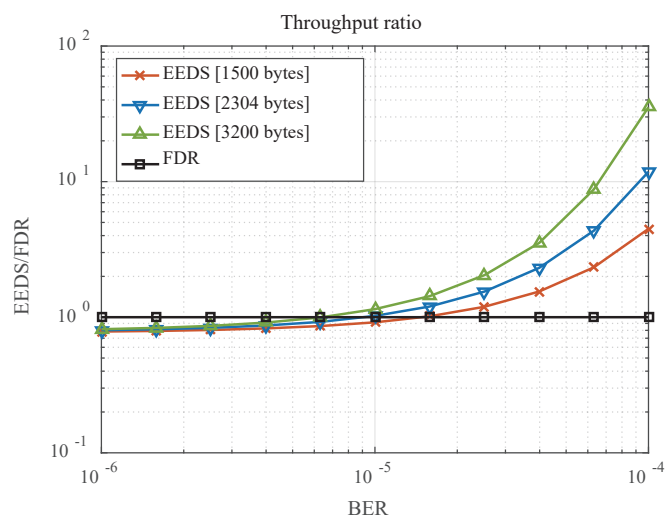

(b)

Fig. 5. (Color online) Throughput ratio for EEDS and FDR: (a) variation in the number of fragments and (b) variation in MSDU size $(N=4)$.

\section{Conclusions}

We presented for FD communication in WSNs, an EEDS that maximizes the spectral efficiency of the FDR system under channel conditions with errors. The EEDS maintains a low error probability during the transmission of the fragments. When an error is detected in any fragment, the EEDS retransmits only the corrupted fragments rather than the entire frame to continue the ongoing transmission of the frame, thereby reducing the amount of time wasted owing to retransmissions. To verify the effectiveness of EEDS, simulations were conducted under various conditions. Results showed that the end-to-end performance of the FDR system with EEDS can be significantly improved under channel conditions with errors.

\section{Acknowledgments}

This research was supported in part by the Leading Human Resource Training Program of Regional Neo Industry through the National Research Foundation of Korea (NRF) funded by the Ministry of Science, ICT and Future Planning (2016H1D5A1910427), by NRF Grant funded by the Korean Government (NRF-2016-Fostering Core Leaders of the Future Basic Science Program/ Global Ph.D. Fellowship Program) (2016H1A2A1908620), and by Institute for Information \& Communications Technology Promotion (IITP) grant funded by the Korea government (MSIP) (No. B0126-15-1076, "Development of non-powered technology combined with ambient RF energy harvesting and backscatter data transfer").

\section{References}

1 A. Sabharwal, P. Schniter, D. Guo, D. W. Bliss, S. Rangarajan, and R. Wichman: IEEE J. Sel. Areas Commun. 32 (2014) 1637.

2 X. Xie and X. Zhang: Proc. INFOCOM (IEEE, New York, 2014) p. 253.

3 M. Jain, J. I. Choi, T. Kim, D. Bharadia, S. Seth, K. Srinivasan, P. Levis, S. Katti, and P. Sinha: Proc. MOBICOM (ACM, New York, 2011) p. 301.

4 S. Sen, R. R. Choudhury, and S. Nelakuditi: CSMA/CN: Proc. MOBICOM (ACM, New York, 2010) p. 25. 